Revista de Psicología de la PUCP. Vol. XIX, 1, 2001

\title{
Relaciones entre felicidad, género, edad y estado conyugal
}

\author{
Reynaldo Alarcón' \\ Universidad Ricardo Palma
}

Se examinaron las presuntas relaciones entre felicidad, género, edad y estado conyugal; asimismo, se determinaron los niveles de felicidad que con mayor frecuencia experimentan las personas. Se trabajó con 163 sujetos de clase media, solteros y casados, de 20 a 60 años, quienes absolvieron la Escala de Satisfacción con la Vida de Diener más un ítem que indagó los niveles de felicidad. Los resultados señalan que no existen diferencias significativas entre las puntuaciones medias de felicidad de varones y mujeres. Según la edad, el único contraste significativo se encontró entre 30 y 50 años y las medias más elevadas correspondieron a los 50 y 60 años. Se halló que las personas casadas son más felices que las solteras. Finalmente, el mayor número de sujetos reportó sentirse feliz y los demás niveles agruparon muy pocas frecuencias. Palabras clave: felicidad, género, edad, estado conyugal, relaciones, niveles de felicidad.

\section{Relationships between happiness and gender, age and marital status}

The present research examines the relationships between happiness and variables of gender, age and marital status as well as the degrees of happiness most frequently experienced by people. The sample was constituted by 163 males and females, between the ages of 20 and 60 years, single and married, and from middle class strata. They were administered the Scale of Satisfaction with Life, developed by Diener, with and added item to measure the degrees of happiness. There is no significant statistically difference between genders; according to age the only significant contrast was between 30 and 50 years, with the notation that the highest means corresponded to ages 50 and 60 years old; married people were found to be happier than single ones. In general, the majority reported feeling happy, the other degrees contained very few frecuencies.

Keywords: happiness, gender, age, conjugal state, relationships, degree of happiness.

1. Doctor en Filosofía y Psicología. Es profesor emérito de la Universidad Nacional Mayor de San Marcos, profesor principal de la Universidad Ricardo Palma y de la Escuela de Postgrado de la Universidad Femenina. Ha publicado numerosos trabajos de investigación empírica y artículos teóricos sobre temas psicológicos de su especialidad. Es miembro del Comité Editorial de la Revista Latinoamericana de Psicología (Bogotá, Colombia), de la Junta de Consultores Editoriales de la Revista Interamericana de Psicología (San Juan, Puerto Rico) y miembro del Consejo Asesor de Cuadernos Argentinos de Historia de la Psicología. Correo electrónico: rcalarcon@terra.com.pe. 

La felicidad es, a menudo, definida como el estado subjetivo de estar bien ("subjetive well-being", SWB) o, también, sentir satisfacción con la vida (Diener, Emmon, Larsen y Griffin, 1985; Diener, Sandvik, Pavot y Fujita, 1992). Este estado de la persona deriva del hecho de experimentar afectos positivos; ciertamente, la felicidad es generada por experiencias gratificantes que promueven sentimientos agradables o emociones positivas que se viven internamente y que, por lo general, tienen un correlato objetivo. Las expresiones de alegría, placer, buen humor, satisfacción, placidez, y otras similares son manifestaciones de felicidad. Los afectos, positivos y negativos, tienen decidida importancia en la felicidad, al punto que algunos autores la definen por ellos (Mroczek y Kolarz, 1998). En un trabajo anterior nosotros encontramos una relación altamente significativa entre SWB y frecuencia de afectos positivos (Alarcón, 2000).

El estado subjetivo de bienestar es una conducta incentivada por una variedad de factores que los psicólogos en recientes décadas buscan identificar. Entre estos se encuentran variables sociodemográficas como género, edad, estado matrimonial, ingreso económico, recursos materiales y las interacciones de unas con otras, que explicarían algunas de las diferencias de felicidad entre los sujetos. Myers y Diener (Lu, 2000) estiman que la felicidad no depende totalmente de factores externos, sino que eventualmente guarda correspondencia con el género, la edad, el estatus socioeconómico y la raza. Wood, Rhodes y Whelan (1989) efectuaron un meta-análisis de 93 estudios y encontraron diferencias significativas de felicidad según el género: las mujeres eran más felices que los hombres. Similar hallazgo ha reportado Mookherjee (1997): las mujeres estaban más satisfechas con la vida que los hombres. Lu (1996) halló en Taiwan que las mujeres estaban más felices que los varones en sus relaciones sociales y vida medioambiental. Francis (1999) administró el Inventario de Felicidad de Oxford (OHI) y la forma breve del Eysenck Personality Questionaire 
a estudiantes varones y mujeres en los que encontró diferencias fundadas en el género. Las puntuaciones del OHI estaban positivamente relacionadas con la extraversión y negativamente correlacionadas con el neuroticismo. Estos resultados apoyan el punto de vista que sostiene que la felicidad puede conceptuarse como "extraversión estable" según el autor.

Las variables género, estado conyugal y felicidad han sido analizadas simultáneamente, involucrando además una amplia gama de otros factores. Russell y Wells (1994) examinaron la importancia relativa de diecisiete predictores de felicidad en 1207 matrimonios. El predictor más fuerte de felicidad conyugal fue la calidad del matrimonio y, en menor grado, el neuroticismo; este influye indirectamente a través de sus efectos sobre la calidad del matrimonio. De los demás predictores, observan que los niños y la familia intervienen directamente en la felicidad. Roger y White (1998) reportaron que la satisfacción matrimonial es significativamente alta en padres y madres casados que tienen hijos biológicos; la satisfacción se incrementa con la calidad del matrimonio; y hay una relación estadísticamente significativa entre la felicidad de los esposos y la felicidad matrimonial. Lu y Lin (1998) correlacionaron el "estar subjetivamente bien" con cuatro roles familiares: esposos, padres, hijos y trabajadores, cruzándolos con cuatro etapas del desarrollo familiar: recién casados sin hijos, casados con hijos menores, casados con hijos adolescentes y casados con hijos adultos. El rol de esposo fue el más sobresaliente en los recién casados sin hijos; el papel de trabajador resultó más importante en los casados con hijos pequeños, en tanto que el rol de trabajador y el rol filial resultaron sobresalientes en los padres con hijos adolescentes. Más adelante, Lu (2000) estudió la armonía familiar en los roles familiares antes señalados y los correlacionó con el SWB en 111 matrimonios de Taiwan. Observó que los maridos se comprometen más con el rol de trabajador y las esposas con el rol maternal; las discrepancias conyugales influyen en el sentirse bien y en la felicidad de los esposos. 
Se ha asociado la felicidad matrimonial con la raza de las personas. Timmer, Veroff y Hatchett (1996) encontraron muchas similitudes entre matrimonios de negros y de blancos, aunque las parejas negras mostraron mayor pertenencia a su familia de procedencia; destacan la importancia de la etnicidad y el contexto estructural, incluido el estrés, para comprender la felicidad de los matrimonios. Aldous y Ganey (1999) han reportado que los matrimonios entre blancos son más felices que los negros y que las mujeres blancas se muestran más contentas que los hombres de su raza. También hallaron que la familia y otros lazos interpersonales eran más importantes para la felicidad de las mujeres blancas que para los varones de su etnia. La satisfacción matrimonial contribuyó más a la satisfacción con la vida de mujeres casadas negras que de los hombres casados de su misma raza. Rank y Davis (1996) solicitaron a parejas de negros y blancos que consideraran lo que serían sus vidas si se separaran. Las esposas y maridos negros coincidieron en señalar que su felicidad fuera del matrimonio sería más alta, respuestas opuestas dieron las parejas blancas. Parte de la explicación de este hecho se debe a que las parejas negras perciben que otros aspectos de sus vidas no serían dañados por el divorcio, como sucedería en los matrimonios de blancos. Estos resultados son consistentes con las tasas más altas de disolución matrimonial y bajas tasas de matrimonios y segundas nupcias en la comunidad negra de USA según comentan los autores. Veroff, Douvan, Orbuch y Acitelli (1998) han estudiado algunos factores que contribuyen a obtener matrimonios estables en parejas negras y blancas. Encuentran que el rol del género está asociado a la felicidad matrimonial; las expectativas del género constituyen las bases sociales de la felicidad de la pareja. Asimismo, el equilibrio afectivo de la pareja y las gratificaciones mutuas contribuyen a la estabilidad matrimonial. De manera contraria, las experiencias negativas y la afirmación del ego en las relaciones actúan como factores críticos. Riehl-Emde y Willi (1999) administraron un cuestionario a parejas normales y a las que acudían a psicoterapia. Los resultados colocan en seria duda la hipótesis sobre diferencias entre 
géneros en estar bien, satisfacción en las relaciones y empatía de la pareja. Afirman que las diferencias entre cónyuges, a menudo, son sobreestimadas. Hintikka, Koskela, Kontula, Koskela y Viinamaeki (1999) han estudiado la relación entre la felicidad en el matrimonio y los desórdenes mentales. Entre sus hallazgos pueden señalarse que los hombres y mujeres que son infelices en sus matrimonios sufren desórdenes mentales comunes más a menudo que las parejas felices; hombres y mujeres de matrimonios infelices se encuentran en riesgo significativamente más alto de desórdenes mentales, comparados con aquellos que tienen un matrimonio feliz. Sin embargo, anotan que el matrimonio per se no está asociado a desórdenes mentales.

En cuanto a las relaciones entre felicidad y edad, Mroczek y Kolarz (1998) examinaron las respuestas de 2727 hombres y mujeres de 25 a 74 años para determinar el efecto de la edad sobre la felicidad. El hallazgo más destacable fue que las personas de mayor edad informaron ser más felices, especialmente las que estaban casadas; tenían menos emociones negativas en comparación con los adultos jóvenes. Los autores señalan que otras investigaciones han observado que los sujetos de mayor edad regulan mejor sus emociones, tienden a maximizar los afectos positivos y minimizar los negativos. Los participantes jóvenes reportaron mayor frecuencia de emociones negativas, sentimientos de tristeza, nerviosismo y desesperanza. Lu, Shih, Lin y Ju (1997) han encontrado que la edad y el género tienen efectos indirectos sobre la felicidad a través del soporte social y la extraversión. El soporte social, según los autores, es un importante mediador y tiene una fuerte asociación con la felicidad. Diener y Such (1998) revisaron un amplio número de investigaciones internacionales sobre la relación entre edad y bienestar subjetivo. Informan que la satisfacción con la vida no declina con la edad aunque sí lo hace la intervención de variables como el matrimonio y el ingreso económico; también observan que los afectos positivos tienden a bajar a través de la cohorte de edades. Según Myers (2000), mucha gente, a nivel mundial, asegura estar moderadamente feliz, independientemente de la edad y el género. 
La revisión de la literatura sobre el tema que nos ocupa no muestra correspondencia unívoca en los resultados respecto de las relaciones o influencias entre felicidad, género, edad y estado conyugal. Al parecer, es indispensable mayor información empírica que contribuya a esclarecer la acción de las variables mencionadas sobre la felicidad. El presente trabajo busca contribuir a tal propósito. Tiene como problemas: (a) determinar si la felicidad, conceptuada como estar subjetivamente bien, guarda relación con el género; (b) si el estado conyugal está asociado a la felicidad; (c) si entre edad y felicidad existe nexo que los relacione; y (d) determinar los niveles de felicidad que con mayor frecuencia experimentan los sujetos.

\section{Metodología}

\section{Participantes}

La muestra estuvo integrada por 163 personas: 81 varones y 82 mujeres; se formaron cinco grupos de edades -20, 30, 40, 50 y 60 años- donde cada uno de ellos tenía entre 31 y 35 miembros. Cada grupo de edad comprende individuos que no pasan el límite de cada década. Por ejemplo, el grupo de 20 años lo integraban jóvenes de 20 a 29 años. La variable estado conyugal comprendió 56 solteros y 92 casados. No se tomó personas de otro estado civil debido a su escasa frecuencia. Desde el punto de vista socioeconómico, los participantes fueron de clase media y media alta de la ciudad de Lima.

\section{Instrumentos}

Utilizamos la Escala de Satisfacción con la Vida de Diener. Los valores psicométricos de esta escala fueron evaluados anteriormente por el presente autor (Alarcón, 2000). Se obtuvo el Alfa de Cronbach $=.528$; según el Método de Mitades de Spearman-Brown, corregido, se obtuvo $r=.647$; y mediante el procedimiento de Guttman split-half , $r=581$. Las correlaciones ítem - test arrojaron una me- 
dia de $r=.337 ; p<.001$. La validez de constructo fue evaluada mediante análisis factorial. Además de la Escala, se utilizó un ítem que evaluó el grado de felicidad que por lo general experimentaban las personas examinadas, con cuatro alternativas: muy feliz, feliz, poco feliz, nada feliz. Las respuestas a este ítem se correlacionaron con la Escala de Diener y se obtuvo un coeficiente Rho $=.430 ; \mathrm{p}<.01$ (para dos colas).

\section{Procedimiento}

Las pruebas fueron administradas en forma colectiva a grupos de estudiantes universitarios de 20 a 30 años. Las personas de las demás edades fueron ubicadas en contextos familiares. Contribuyeron a la aplicación de las pruebas algunos de los estudiantes de talleres de investigación, de la Universidad Ricardo Palma y estudiantes de Maestría en Psicología de la Escuela de Postgrado de la Universidad Femenina.

\section{Resultados}

Comparaciones entre las puntuaciones obtenidas en la Escala de Satisfacción con la vida, el género, la edad y el estado conyugal.

Para determinar la influencia que el género ejerce sobre la felicidad, se han comparado las medias obtenidas por varones y mujeres en la Escala de Satisfacción con la Vida. La media para hombres fue de 17.69 y para mujeres 17.76, el test de Student entre ambas medias fue $t=-0.136$, valor que carece de significación al nivel $p=.05$, para dos colas. Este resultado indica que el género es una variable no determinante de la felicidad o, para decirlo en otros términos, hombres y mujeres suelen ser igualmente felices, por lo menos entre sujetos de estatus socioeconómico medio y medio alto que corresponde a la muestra examinada. 
Para determinar la presunta relación entre edad y felicidad, se formaron cinco grupos de edades 20,30,40,50 y 60 . El Cuadro 1 presenta los resultados del análisis unidireccional de varianza, el valor de la razón $F=2.782(p<.05)$ indica que existen diferencias entre las edades; para establecer entre qué pares de edades las diferencias son significativas se utilizó la prueba de Tukey HSD; el único contraste significativo se encontró entre 30 y 50 años, la media para 30 años fue 16.81 y para 50 años de 19.26 , la diferencia de 2.45 fue significativa a $p<.05$. Entre las demás edades las diferencias carecen de significación estadística. No obstante, se puede observar que las medias de felicidad más elevadas corresponden a las edades mayores $(50$ años $=19.26$ y 60 años $=18.09)$. En rigor, los datos solo permiten inferir la escasa influencia que ejerce la edad sobre el sentirse feliz; así también, pone en duda la creencia difundida que las personas mayores son menos felices que las personas más jóvenes.

\section{Cuadro 1}

Análisis de varianza entre los resultados obtenidos según edad en la Escala de Satisfacción con la Vida

\begin{tabular}{|lrrrrr|}
\hline $\begin{array}{l}\text { Fuente de } \\
\text { Variación }\end{array}$ & SC & gI & CM & $F$ & Sig. \\
\hline Entregrupos & 119.37 & 4 & 29.84 & $2.78^{*}$ & .029 \\
Intragrupos & 1695.20 & 158 & 10.73 & & \\
Total & 1814.57 & & & & \\
\hline
\end{tabular}

Nota. Los grupos de edad son $20,30.40 .50$ y 60 años.

$* p<.05$

La tercera variable fue el estado conyugal de los sujetos, se formaron dos grupos: solteros y casados. Las medias obtenidas en la Escala de Diener fueron: solteros $M=16.95$, casados $M=18.38$. La media de casados es más elevada que la de solteros. La diferencia entre ambas medias fue significativa a $p<.05$ según el Test $t$ de Student. Este resultado se puede interpretar en el sentido que las personas casadas son más felices que las solteras. Es pertinente señalar 
que en el grupo de casados no existen diferencias significativas entre las medias de felicidad según el sexo. El grupo de varones obtuvo $M=18.27$ y las mujeres $M=18.53$. Al parecer, lo que determina la felicidad, según estas variables, es el estado matrimonial y no el género de los individuos. Es de presumir que las personas casadas, hombres y mujeres hayan tenido, en el curso de su vida matrimonial, experiencias afectivas satisfactorias y gratificantes.

\section{Efectos de los factores género, edad y estado conyugal y sus valo- res, sobre la variable felicidad.}

Con la finalidad de verificar el efecto que cada factor y sus valores, más la interacción que los factores principales puedan ejercer sobre la variable felicidad, hemos sometido los datos a un análisis factorial de varianza $(2 \times 5 \times 2)$. Los resultados del Cuadro 2 indican que solamente es significativa la interacción estado conyugal $x$ edad, de lo que se infiere que la diferencia advertida entre casados y solteros es significativa en tanto se relaciona con la variable edad. En efecto, la media obtenida por varones casados de 50 y 60 años fue de 19.30, en tanto que la de varones casados de 30 y 40 años alcanzó 17.18. El grupo de mujeres casadas de 50 y 60 años obtuvo una media de 18.92 y el grupo de casados de 20, 30 y 40 años alcanzó 16.94 . Ya hemos señalado anteriormente que los solteros, en términos generales, obtienen medias de felicidad más bajas que los casados. En suma, la edad y sus valores y el estado conyugal y los suyos interactúan ofreciendo una razón $F$ significativa. Carecen de significación estadística la interacción género $\mathrm{x}$ estado conyugal, género $\mathrm{x}$ edad y la interacción de las variables género $\mathrm{x}$ estado conyugal $\mathrm{x}$ edad.

El coeficiente de correlación múltiple, $R^{2}$ ajustado $=.167$, indica que las variables independientes, en conjunto, y las interacciones consideradas en el modelo explican el $16.7 \%$ de la varianza total de la variable felicidad. Obsérvese que $R^{2}=.285$ es más elevado, lo cual aumentaría la magnitud de la varianza explicada. Hemos preferido utilizar $R^{2}$ ajustado para controlar influencias debido al tamaño de 
la muestra. De los coeficientes Eta ${ }^{2}$ sobresale la interacción estado conyugal $x$ edad que junto con sus niveles explican el $13.8 \%$ de la varianza de la variable dependiente felicidad. En suma, es modesto el aporte para explicar la felicidad de las tres variables sociodemográficas que venimos estudiando.

\section{Cuadro 2}

Análisis factorial de varianza entre los factores género, edad, estado conyugal y sus niveles

\begin{tabular}{|lrrrrrr|}
\hline $\begin{array}{l}\text { Fuente de } \\
\text { Variación }\end{array}$ & \multicolumn{1}{c}{$\mathrm{SC}$} & $\mathrm{gl}$ & $\mathrm{CM}$ & $F$ & Sig. & Eta $^{2}$ \\
\hline Modelo corregido & $517,952^{a}$ & 23 & 22.520 & 2.414 & .001 & .285 \\
Intercepción & 16093.833 & 1 & 16093.833 & 1725.282 & .000 & .925 \\
Género & 16.598 & 1 & 16.598 & 1.779 & .184 & .013 \\
Estado & 32.765 & 2 & 16.382 & 1.756 & .177 & .025 \\
Edad & 52.217 & 4 & 13.054 & 1.399 & .237 & .039 \\
Género x estado & 45.740 & 2 & 22.870 & 2.452 & .090 & .034 \\
Género x edad & 86.313 & 4 & 21.578 & 2.313 & .061 & .062 \\
Estado x edad & 207.465 & 7 & 29.638 & $3.177^{*}$ & .004 & .138 \\
Gén. x est. x edad & 7.063 & 3 & 2.354 & .252 & .860 & .005 \\
Error & 1296.625 & 139 & 9.328 & & & \\
Total & 53019.000 & 163 & & & & \\
Total corregido & 1814.577 & 162 & & & & \\
\hline
\end{tabular}

Nota. La variable dependiente es felicidad.

$" R^{2}=.285$ ( $R^{2}$ ajustado $=.167$ )

$* p<.05$

\section{Los niveles de felicidad, según género, edad y estado conyugal.}

La felicidad, como algunas otras conductas, se manifiesta en grados de intensidad que pueden variar entre la plena felicidad hasta la ausencia de ella. Un ítem de nuestra prueba, como se dijo ut supra, permite obtener un informe directo de este hecho. En el Cuadro 3 se encuentran los resultados del análisis loglineal, model selection, a que fueron sometidos los datos según el género. Puede verse que el mayor número de frecuencias observadas (FO) corresponden a la casilla 
feliz, tanto para varones como para mujeres; muy pocas personas ocupan la casilla nada feliz; también se advierte que hay un mayor número de hombres muy felices (13\%), contra un $4 \%$ de mujeres. En el mismo cuadro aparecen las frecuencias esperadas, los residuales y los residuales estandarizados. La probable asociación o independencia entre las variables felicidad y género se ha verificado mediante $X^{2}$ y la razón de verosimilitud de $X^{2}$ (RV $X^{2}$ ). Estos valores carecen de significación a $p<.05$, es decir, ambas variables no están asociadas, confirmando el hallazgo anterior que el género de los individuos no es una variable que afecte la felicidad de las personas.

\section{Cuadro 3}

Análisis loglineal model selection de las variables género y niveles de felicidad

\begin{tabular}{|lrrrr|}
\hline Variables & FO & FE & Residual & R. Stad. \\
\hline Hombre & & & & \\
Nada feliz & 1.0 & 2.0 & -.99 & -.70 \\
Poco feliz & 8.0 & 10.9 & -2.93 & -.89 \\
Feliz & 59.0 & 59.6 & -.63 & -.08 \\
Muy feliz & 13.0 & 8.4 & 4.55 & 1.57 \\
& & & & \\
Mujer & 3.0 & 2.0 & .99 & .70 \\
Nada feliz & 14.0 & 11.1 & 2.93 & .88 \\
Poco feliz & 61.0 & 60.4 & .63 & .08 \\
Feliz & 4.0 & 8.6 & -4.55 & -1.56 \\
Muy feliz & & & & \\
\hline
\end{tabular}

Nota. Razón de Verosimilitud de $X^{2}=7.7477 g l=3 p=.052 \mathrm{~ns}$.

$$
X^{2}=7.4285 g l=3 p=.059 \text { ns. }
$$

En el Cuadro 4 se reportan los resultados del análisis loglineal, model selection; de la variable edad, con cinco niveles y la variable felicidad. Al observar la distribución de las FO se advierte que en el nivel feliz se agrupa el mayor número de sujetos de los cinco grupos de edades en cantidades aproximadamente iguales; los niveles extremos, nada feliz y muy feliz, concentran muy pocas frecuencias, aunque en este último nivel haya un número comparativamente mayor 
Relaciones entre felicidad, género, edad y estado conyugal

de sujetos de 50 y 60 años. Los valores de $X^{2}$ y de RV $X^{2}$ que se encuentran al pie del cuadro indican que las variables felicidad y edad no están significativamente asociadas.

\section{Cuadro 4}

Análisis Loglineal model selection de las variables edad y niveles de felicidad

\begin{tabular}{|lrrrr|}
\hline Variables & FO & FE & Residual & R. Std. \\
\hline Nada feliz & & & & \\
20 años & 1.0 & .9 & .14 & .15 \\
30 & 1.0 & .8 & .21 & .24 \\
40 & .0 & .8 & -.76 & -.87 \\
50 & .0 & .8 & -.76 & -.87 \\
60 & 2.0 & .8 & 1.17 & 1.28 \\
Poco Feliz & & & & \\
20 años & 6.0 & 4.7 & 1.28 & .59 \\
30 & 4.0 & 4.3 & -.32 & -.15 \\
40 & 8.0 & 4.2 & 3.82 & 1.87 \\
50 & 2.0 & 4.2 & -2.18 & -1.07 \\
60 & 2.0 & 4.6 & -2.59 & -1.21 \\
Feliz & & & & \\
20 años & 27.0 & 25.8 & 1.23 & .24 \\
30 & 25.0 & 23.6 & 1.44 & .30 \\
40 & 20.0 & 22.8 & -2.82 & -.59 \\
50 & 23.0 & 22.8 & .18 & .04 \\
60 & 25.0 & 25.0 & -.03 & -.01 \\
Muy feliz & & & & \\
20 años & 1.0 & 3.7 & -2.65 & -1.39 \\
30 & 2.0 & 3.3 & -1.34 & -.73 \\
40 & 3.0 & 3.2 & -.23 & -.13 \\
50 & 6.0 & 3.2 & 2.77 & 1.54 \\
60 & 5.0 & 3.5 & 1.45 & .77 \\
\hline
\end{tabular}

Nota. Razón de Verosimilitud de $X^{2}=16.915 \mathrm{gl}=12 \mathrm{p}=.153 \mathrm{~ns}$.

$$
X^{2}=15.619 g l=12 p=.209 \mathrm{~ns} \text {. }
$$

Para terminar estos análisis, el Cuadro 5 exhibe los resultados correspondientes a las variables estado conyugal (soltero, casado) y felicidad con sus cuatro niveles. De manera similar a lo observado en las distribuciones de los Cuadros 3 y 4 , aquí también el mayor número 
de sujetos ( $75 \%$ de $\mathrm{n}=148$ ) se agrupan en el nivel feliz; en el nivel muy feliz el número de casados es superior al de solteros. La magnitud de $X^{2}=15.69$ es significativa a $p<.01$, lo mismo que la RV $X^{2}=16.39$. Estos valores indican que las variables felicidad y estado conyugal están significativamente asociadas; dicho en otros términos, el estado matrimonial de las personas influye en sus niveles de felicidad.

De los tres análisis loglineales, model selection, puede inferirse que los individuos tienden a agruparse con mayor frecuencia en torno al nivel feliz según el género, la edad y el estado conyugal. Muy pocas personas manifiestan sentirse nada feliz y muy feliz, excepto cuando se toma la variable estado conyugal donde hay un $15.21 \%$ de sujetos casados que expresan sentirse muy felices. Asimismo, se ha podido establecer que entre grados de felicidad, género y edad no existe nexo asociativo significativo y que las variables felicidad y estado conyugal están fuertemente asociadas.

\section{Cuadro 5}

Análisis loglineal model selection de las variables estado conyugal y niveles de felicidad

\begin{tabular}{|lrrrr|}
\hline Variables & FO & FE & Residual & R. Std. \\
\hline $\begin{array}{l}\text { Nada feliz } \\
\text { soltero }\end{array}$ & 2.0 & 2.0 & .00 & .00 \\
casado & 2.0 & 2.0 & .00 & .00 \\
$\begin{array}{l}\text { Poco feliz } \\
\text { soltero }\end{array}$ & 10.0 & 8.0 & 2.0 & .71 \\
casado & 6.0 & 8.0 & -2.00 & -.71 \\
$\begin{array}{l}\text { Feliz } \\
\text { soltero }\end{array}$ & 41.0 & 55.5 & -14.50 & -1.95 \\
casado & 70.0 & 55.5 & 14.50 & 1.95 \\
Muy feliz & & & & -1.89 \\
soltero & 3.0 & 8.5 & -5.50 & 1.89 \\
casado & 14.0 & 8.5 & 5.50 & \\
\hline
\end{tabular}

Nota. Razón de Verosimilitud de $X^{2}=16.3989 g l=4 \quad p=.003 \quad p<.01$

$$
X^{2}=15.6942 g l=4 \quad p=.003 \quad p<.01
$$


Relaciones entre felicidad, género, edad y estado conyugal

\section{Discusión}

Vamos a comentar los resultados hallados en este trabajo empezando por la variable género. Encontramos que no existen diferencias significativas al comparar las medias aritméticas obtenidas por varones y mujeres en la Escala de Satisfacción con la Vida. Ambos géneros son igualmente felices. Este resultado fue verificado mediante el análisis loglineal que mostró que las variables felicidad y género no están asociadas. Nuestros resultados no guardan correspondencia con los hallazgos de Wood, Rhodes y Whelan (1989) y Mookherjee (1997) quienes han encontrado que las mujeres son más felices que los hombres. Lu (1996) descubrió que las mujeres están más satisfechas que los hombres en algunos aspectos de la vida, como por ejemplo, las relaciones sociales, en tanto que los varones se interesan más por el trabajo. También se sostiene el punto de vista que las fuentes de felicidad y de satisfacción con la vida pueden ser diferentes para mujeres y varones (Argyle, en Lu, 2000).

Nos parece que en las relaciones entre felicidad y género influyen factores culturales. Con esto queremos señalar que los individuos se comportan socialmente de acuerdo con las pautas de su sociocultura, la cual provee patrones de comportamiento que se transmiten a través de generaciones, aunque pueden variar con el paso de los años. Actitudes, creencias, estereotipos sexuales y asignación de roles al hombre y a la mujer corresponden a estructuras mentales modeladas por la sociocultura en la que viven los individuos. En sociedades tradicionales, de organización patriarcal, como las que subsisten en muchos países de América Latina hay una secuencia histórica de discriminación de la mujer junto a un pronunciado machismo. De estos estilos de vida no escapa el Perú, aunque solo en recientes años se ha puesto interés en estos problemas. Psicólogos que trabajan en programas de intervención de violencia familiar han observado que en las familias violentadas prima un tipo de relación rígida y jerárquica, que las decisiones son tomadas por el hombre y que existe una marcada desigualdad entre los géneros, siendo el hombre el que ejerce 
violencia sobre su pareja. Anotan que el 53\% de las mujeres atendidas por maltrato pertenecen al nivel socioeconómico bajo, en su mayoría casadas (Aguilar, Enriquez, Hidalgo y Muente, 2000). También se ha reportado que en las comisarias de mujeres de Lima se registra un total de 32,030 denuncias de violencia por mes (Junco, 2000). En el marco de estas circunstancias sociales, nuestros resultados aparecen optimistas puesto que señalan que varones y mujeres son igualmente felices. Probablemente estos hallazgos podrían variar de incluirse en la muestra individuos de estrato socioeconómico bajo. Además, hay evidencias que los miembros de los sectores deprivados de la sociedad presentan, con mayor frecuencia, estados de depresión, pesimismo y tristeza cuando se les compara con individuos de sectores medios y altos (Alarcón, 1968).

Los resultados entre edad y felicidad encontrados en este trabajo guardan correspondencia con los obtenidos por otros investigadores. Según nuestros datos, la satisfacción con la vida no declina con la edad, entre 20 a 60 años; aunque no hemos encontrado una asociación lineal ascendente entre ambas variables. Diener y Suh (1998) analizaron investigaciones internacionales sobre el tema y concluyeron que la felicidad no declina con la edad. También hemos observado que las puntuaciones medias más altas de felicidad fueron obtenidas por sujetos de 50 y 60 años. Un hallazgo similar obtuvieron Mroczek y Kolarz (1998). Ellos reportaron que las personas viejas informaron sentirse felices, especialmente los hombres casados (el rango de edades investigadas fue de 25 a 74 años). Estos resultados son importantes toda vez que, muy a menudo, se ha supuesto todo lo contrario, ya que el adulto mayor, con la declinación de su salud, el deceso - que con frecuencia ocurre- del cónyuge y de los amigos, más otros problemas que acarrea la vejez, podría presentar más elementos que generen infelicidad.

Se han propuesto algunas hipótesis para explicar este hecho. En efecto, se sostiene que los adultos mayores regulan mejor sus emociones, aprenden a maximizar los afectos positivos y a minimizar los 
afectos negativos. Debe recordarse que los afectos positivos guardan una estrecha correlación con la felicidad. Su importancia es tal que algunos autores, como Mroczek y Kolarz (1998), definen la felicidad por afectos positivos y negativos. Se sostiene, asimismo, que los roles de los afectos y de la cognición se reestructuran con la mayoría de edad, permitiendo una gran cohesión entre ambos, lo que facilita regular las emociones, a la vez que ofrece la madurez necesaria para maximizar los afectos positivos y minimizar los negativos (Carstensen, 1991, 1995; Labovic-Vief y Blanchard-Fields, 1982).

En cuanto a la relación entre estado conyugal y felicidad, hallamos que las personas casadas son más felices que las solteras. Observamos también que no habían diferencias significativas entre las medias de felicidad de varones y mujeres. Estos resultados nos ha llevado a inferir que lo que determina la felicidad es el estado matrimonial y no el género de los sujetos. Puesto que no fue problema del presente trabajo indagar por la felicidad matrimonial, es de presumir, a la luz de otras investigaciones, que el mayor número de personas, varones y mujeres examinadas, disfrutaban de satisfacción matrimonial, la cual es generada por un amplio número de variables, según dimos cuenta en la revisión de la literatura sobre el tema.

Respecto del problema de la intensidad de la felicidad que, en términos generales, experimentaban las personas, nuestros resultados son constantes según el género, la edad y el estado conyugal: el mayor número de sujetos manifestó sentirse feliz. Muy pocas frecuencias obtuvieron los niveles muy feliz y nada feliz. Mroczek y Kolarz (1998) comentan que Inglehart utilizó dos bases de datos, el Euro - Barometer Survey y el World Value Survey, que fueron administrados en dieciséis naciones. Encontró que el porcentaje de gente que informó estar muy feliz fue relativamente constante a través de un amplio rango de edades (15 a 68 años). También observó que, en algunos países, la felicidad decrecía entre los más viejos; en otras naciones aumentaba, en tanto que en algunas otras no existían diferencias a través de las edades. 
Combinando estos resultados transnacionales, puede inferirse que la edad no tiene efectos definidos sobre la felicidad.

Para terminar, también buscamos determinar el efecto de cada factor (género, edad y estado conyugal), los valores de cada uno de ellos y sus interacciones sobre la felicidad. El análisis factorial de varianza puso en evidencia que solamente era significativa la interacción estado conyugal por edad, deduciéndose que la diferencia entre casados y solteros es significativa en tanto se relaciona con los valores de la variable edad. El coeficiente $R^{2}$ ajustado indicó que las tres variables, en conjunto, solamente explican el $16.7 \%$ de la varianza de la variable felicidad. Algunos investigadores (Ryff, 1989; Diener, 1994; Myers y Diener, 1995) atribuyen a las variables sociodemográficas, como las analizadas en la presente investigación, una modesta influencia pues explican una pequeña porción de la varianza de la variable felicidad, tal como nosotros lo hemos encontrado.

\section{Referencias}

Aguilar, M. Y, Enriquez, D. E., Hidalgo, T. y Muente, M. (2000). Taller para mujeres víctimas de violencia familiar: Una propuesta. "Más me pegas, menos te quiero". Revista Peruana de Psicología, 5, $47-65$.

Alarcón R. (1968). Psicología, pobreza y subdesarrollo. Lima: Consejo Nacional de Ciencia y Tecnología.

Alarcón, R. (2000). Variables psicológicas asociadas con la felicidad. Persona, 3, 147-157.

Aldous, J. y Ganey, R. F. (1999). Family life and pursuit of happiness: The influence of gender and race. Journal of Family Issues, 20. 155-180

Carstensen, L. L. (1991). Selective theory: Social activity in life-span contex. Annual Review of Gerontology and Geriatrics, 11, 195-217. 
Carstensen, L. L. (1995). Evidence for life-span theory of socioemotional selective. Current Directions in Psychologilcal Science, 4, 151-155.

Diener, E. (1994). Assessing subjetive well-being: Progress and opportunities. Social Indicators Research, 31, 103-157.

Diener, E., Emmon, R. A. , Larsen, R. J. y Griffin, E. (1985). The satisfaction with life scale. Journal of Personality Assessment, 49, 7175

Diener, E., Sandvik, E., Pavot, W. y Fujita, F. (1992). Extraversion and subjetive well-being in US. national probability sample. Journal of Research in Personality, 26, 205-215.

Diener, E. y Suh, M. E. (1998). Subjetive well-being and age: An international analysis. En S. K. Warner y L. M. Powell (Eds.), Annual review of gerontology and geriatric (pp. 304-324). Nueva York: Spinger Publishing.

Francis, L. J. (1999). Happiness is a thing called stable extraversion: A further examination of relationship between the Oxford Happiness Inventory and Eysenck's dimensional model of personality and gender. Personality and Individual Differences, 26, 5-11.

Hintikka, J., Koskela, T., Kontula, O., Koskela, K. y Viinamaeki, H. (1999). Men, women, and marriages: Are there differences in relation to mental health? Family Therapy, 26, 213-128.

Junco, J. (2000). Violencia familiar en el Perú. Revista Peruana de Psicología, 5, 81-92.

Labouvie-Vief, G. y Blanchard-Fields, F. (1982). Cognitive aging and psychological growth. Ageing and Society, 2, 183-209.

Lu, L. (1996). Correlates of happiness among the chinese people. Taipei, Taiwan, ROC: National Science Council.

Lu, L. (2000). Gender and conyugal differences in happiness. The Journal of Social Psychology, 140, 132-142.

Lu, L., Shih, J. B., Lin, Y. Y. y Ju, L. S. (1997). Personal and enviromental correlates of happiness. Personality and Individual Differences, $23,453-462$.

Lu, L. y Lin, Y. Y. (1998). Family roles and happiness in adulthood. Personality and Individual Differences, 25, 195-207. 
Mookherjee, H. N. (1997). Marital status and percepción of well-being. The Journal of Social Psychology, 137, 95-105.

Mroczek, D. K. y Kolarz, C. M. (1998). The effect of age on positive and negative affect : A developmental perspective on happiness. Journal Personality and Social Psychology, 75, 1333-1349.

Myers, D. G. (2000). The funds, friends, and faith of happy people. American Psychologist, 55, 56-67

Myers, D. G. y Diener, E. ( 1995). Who is happy? Psychological Science, 6. 10-19.

Rank, M. R. y Davis, L E. (1996). Perceived happiness outside of marriage among Black and White spouses. Family Relations: Journal of Applied Family \& Child Studies, 45, 435-441.

Riehl-Emde, A. y Willi, J. (1999). Is his marriage also her marriage? An old question in a new light. System Familie-Forschung und Therapie, 12 (3),132-138.

Rogers, S. J. y White, L. K. (1998). Satisfaction with parenting: The role of marital happiness, family structure, and parents'gender. Journal of Marriage and the Family, 60. 293-308.

Russell, R. J. H. y Wells, P. A. (1994). Predictors of happiness in married couples. Personality and Individual Differences, 17, 313-321.

Ryff, D. D. (1989). Happiness is everything, or is it? Exploration on the meaning of psychological well-being. Journal of Personality and Social Psychology, 57, 1069-1081.

Timmer, S. G., Veroff, J. y Hatchett, E. (1996). Family ties and marital happiness: The different marital experiencies of Black and White newlywed couples. Journal of Social and Personality Retationships, 13, 335.359.

Veroff, J., Douvan, E., Orbuch, T. L. y Acitelli, L.. K. (1998). Happiness in stable marriages: The early years. En T. W. N. Bradbury (Ed.), The developmental course of marital dysfunction (pp.152-179). Nueva York: Cambridge University Press.

Wood, W., Rhodes, N. y Whelan, M. (1989). Sex differences in positive well-being: A consideration of emotional style and marital status. Psychological Bulletin, 106, 249-264. 